Review Article

\title{
Influence of Gestational Hormones on the Bacteria-Induced Cytokine Response in Periodontitis
}

\author{
Betsaida J. Ortiz-Sánchez $\mathbb{D}^{1},{ }^{1}$ Martha Legorreta-Herrera $\mathbb{D}^{\circ},{ }^{2}$ and Miriam Rodriguez-Sosa $\mathbb{D}^{3}$ \\ ${ }^{1}$ Dental Surgeon Career, Facultad de Estudios Superiores Iztacala, Universidad Nacional Autónoma de México (UNAM), \\ MEX. C.P. 54090, Tlalnepantla, Mexico \\ ${ }^{2}$ Molecular Immunology Laboratory, Facultad de Estudios Superiores, Zaragoza, UNAM, Iztapalapa, \\ Mexico City C.P. 09230, Mexico \\ ${ }^{3}$ Innate Immunity Laboratory, Facultad de Estudios Superiores Iztacala, UNAM, MEX C.P. 54090, Tlalnepantla, Mexico \\ Correspondence should be addressed to Miriam Rodriguez-Sosa; rodriguezm@unam.mx
}

Received 27 July 2021; Revised 23 September 2021; Accepted 27 September 2021; Published 18 October 2021

Academic Editor: Gaetano Isola

Copyright (C) 2021 Betsaida J. Ortiz-Sánchez et al. This is an open access article distributed under the Creative Commons Attribution License, which permits unrestricted use, distribution, and reproduction in any medium, provided the original work is properly cited.

\begin{abstract}
Periodontitis is an inflammatory disease that affects the supporting structures of teeth. The presence of a bacterial biofilm initiates a destructive inflammatory process orchestrated by various inflammatory mediators, most notably proinflammatory cytokines, which are upregulated in the gingival crevicular fluid, leading to the formation of periodontal pockets. This represents a wellcharacterized microbial change during the transition from periodontal health to periodontitis; interestingly, the gestational condition increases the risk and severity of periodontal disease. Although the influence of periodontitis on pregnancy has been extensively reviewed, the relationship between pregnancy and the development/evolution of periodontitis has been little studied compared to the effect of periodontitis on adverse pregnancy outcomes. This review is aimed at summarizing the findings on the pregnancy-proinflammatory cytokine relationship and discussing its possible involvement in the development of periodontitis. We address (1) an overview of periodontal disease, (2) the immune response and possible involvement of proinflammatory cytokines in the development of periodontitis, (3) how bone tissue remodelling takes place with an emphasis on the involvement of the inflammatory response and metalloproteinases during periodontitis, and (4) the influence of hormonal profile during pregnancy on the development of periodontitis. Finally, we believe this review may be helpful for designing immunotherapies based on the stage of pregnancy to control the severity and pathology of periodontal disease.
\end{abstract}

\section{Introduction}

Periodontal disease is an inflammatory condition of periodontal tissues with a heterogeneous aetiology and is one of the most common diseases in the world [1]. This disease affects the gum and supporting tissues of the teeth, alveolar bone, periodontal ligament, and root cementum. Approximately $60 \%$ of the total population has some degree of periodontal disease [2]. In Latin America, this number increases to up to $90 \%$ [3]. The early stage of development of this pathology is called gingivitis and affects only the soft tissues. The severe form, called periodontitis, severely affects periodontal tissues, mainly alveolar bone, with subsequent loss of insertion of dental organs.
The development of periodontal disease and its progression depend on different factors that modulate the host immune response against the biofilm, such as genetic and epigenetic predisposition including hereditary angyoderma [4], social factors, habits (such as tobacco and alcohol use and poor oral hygiene) [5], advanced age [6], and systemic conditions, such as obesity, malnutrition [7], infections (such as HIV/AIDS), osteoporosis and stress [8], type 1 and 2 diabetes [9], and scleroderma disease [10]. Notably, periodontitis affects $23 \%$ of women between 23 and 54 years of age and is present in $56 \%$ of pregnant women [11]. Furthermore, recent evidence suggests that hormonal treatment, the use of hormonal contraceptives, and pregnancy induce clinical, cytological, or microbiological changes in women 
[12], which probably promote the development of this disease.

Inflammatory cytokines are upregulated during pregnancy and increased during ovulation, in early gestation, in term pregnancy, and during delivery [13]. However, it has not been established whether there is any relationship between pregnancy/proinflammatory cytokines and the development of periodontal disease. In this review, we summarize the current knowledge by providing a broad overview of periodontitis and then focusing specifically on recent findings related to the inflammatory response in pregnancy and its possible relationship to the development of periodontitis.

\section{Periodontitis and the Oral Microbiome}

The oral cavity has a dynamic environment that is formed by the oral microbiome with all of its interspecies interactions but also interactions with the oral cavity, creating a symbiotic relationship with the human host [14]. Periodontitis is initiated by polymicrobial synergy, and dysbiosis is modified by numerous risk factors. Competitive and cooperative interspecies interactions of microbial communities can shape the nature and function of the entire microbiome synergism [15]. The subgingival microbiome includes the red complex triad (Treponema denticola, Tannarella Forsythia, and Porphyromonas gingivalis) [16], orange complex triad (Fusobacterium nucleatum, Prevotella intermedia, and Parvimonas micra), Actinobacillus actinomycetemcomitans, Campylobacter rectus, Eikenella corrodens, Bacteroides forsythus [17], Filifactor alocis [18], Peptoanaerobacter stomatis, Firmicutes phylum, Methanobrevibacter oralis [19], C. albicans [20], and human cytomegalovirus and Epstein-Barr virus [21].

The periodontal microbiome is complex and constitutes the cornerstone in the development of periodontal disease. The characteristics of the bacteria themselves are essential in determining the course of the immune response. For example, Porphyromonas gingivalis can modulate the innate inflammatory response [22]. Filifactor alocis also induces oxidative stress and alters the recognition capacity of the inflammatory response by inactivating the complement pathways [23]. Filifactor alocis and other bacteria, such as Porphyromonas gingivalis, are highly invasive and promote dysbiosis of the microbiota [24]; therefore, a pathogenesis model has been proposed in which periodontal disease is initiated due to dysbiosis of the microbiota, called the PSD (polymicrobial synergy and dysbiosis) model [25].

\section{Immune Response in Periodontitis}

Immune cells interact with biofilms when their pattern recognition receptors (PRRs) detect pathogen-associated molecular patterns (PAMPs) present on bacteria. These receptors are expressed on innate immune cells, such as neutrophils, eosinophils, basophils, macrophages $(\mathrm{M} \varphi \mathrm{s})$, monocytes, dendritic cells (DCs), and natural killer (NK) cells, and adaptive immune cells, such as T and B lymphocytes, as well as on nonimmune cells, such as epithelial cells, endothelial cells, and fibroblasts [26]. This PAMP-PPR interaction activates the innate immune response characterized by neutrophil, eosinophil, and basophil recruitment, consequently activating the complement system [27].

This first recognition is characterized by acute inflammation; if biofilm dysbiosis persists, this response develops into chronic inflammation. In this phase, osteoclast activation is favoured. It results in bone resorption, with subsequent degradation of the bone matrix and periodontal ligament fibres by metalloproteinases (MMPs) and the formation of granulation tissue [28] (Figure 1). Thus, in both acute inflammation and chronic inflammation, cytokines, and inflammatory mediator's determinate disease progression factors.

Antigen-presenting cells (APCs), such as DCs, recognize pathogens expressing PAMPs, internalize these pathogens by phagocytosis, and degrade and process pathogenderived antigens, transforming the antigens into small peptides that bind to major histocompatibility complex (HLA) molecules for display on the cell surface. This presentation is accompanied by the expression of the costimulatory molecules CD86 and CD40. DCs migrate to secondary lymphoid tissues (lymph nodes and lymphoid tissue) to present antigens and thus activate $\mathrm{CD} 4^{+} \mathrm{T}$ cells to generate an antigen-specific immune response [29]. CD4+ T cells differentiate into regulatory and effector $\mathrm{T}$ cell subsets: Th1, Th2, Th17, follicular helper $\mathrm{T}$ (Tfh) cells, and regulatory $\mathrm{T}$ cells (Tregs) [30]. The differentiation of Th1 and Th2 cells is mutually antagonistic; Treg and Th17 cells share the same origin and have opposite effects, while Th17 cells cause autoimmunity and inflammation, and Treg cells inhibit these and maintain immune homeostasis.

The activation profile of $\mathrm{CD}^{+} \mathrm{T}$ lymphocytes in periodontal disease varies depending on disease progression. In the initial phase, $\mathrm{CD}^{+} \mathrm{T}$ lymphocytes exhibit a proinflammatory Th-1 profile characterized by the synthesis of macrophage inhibitory factor (MIF), interleukin- (IL-) 2, and interferon- (IFN-) $\gamma$, which promote cellular immunity and the activation of cytotoxic $\mathrm{CD}^{+} \mathrm{T}$ lymphocytes (TCs) and Th-17 cells [31]. Other cytokines, such as IL- $1 \alpha$, IL- $1 \beta$, IL8 , IL-6, and tumour necrosis factor- (TNF-) $\alpha$ produced by monocytes, $M \varphi s$, DCs, and neutrophils, are also produced under these conditions [32]. In addition, endothelial cells, fibroblasts, and osteoclasts produce prostaglandin E2 (PGE2) and granulocyte macrophage colony-stimulating factor (GM-CSF) [33]. Together, these conditions promote the expression of receptor activator of NF- $\kappa \mathrm{B}$ ligand (RANKL), leading to osteoclastogenesis [34].

In the chronic phase of periodontitis, $\mathrm{CD}^{+} \mathrm{T}$ lymphocytes differentiate towards an anti-inflammatory Th2 profile, characterized by the production of IL-4, IL-5, IL-6, and IL10 [35]. This profile favours B lymphocyte activation and subsequent differentiation into IgG-type immunoglobulinproducing plasma cells [36]. In this way, when periodontitis becomes chronic, negative regulation of inflammation through the anti-inflammatory cytokines IL-4, IL-6, IL-10, IL-11, and IL-13 becomes predominant [37]. This immunoregulation is a complex phenomenon involving mediators such as RANKL-DCs, favouring the activation of $\mathrm{CD} 4^{+}$ 


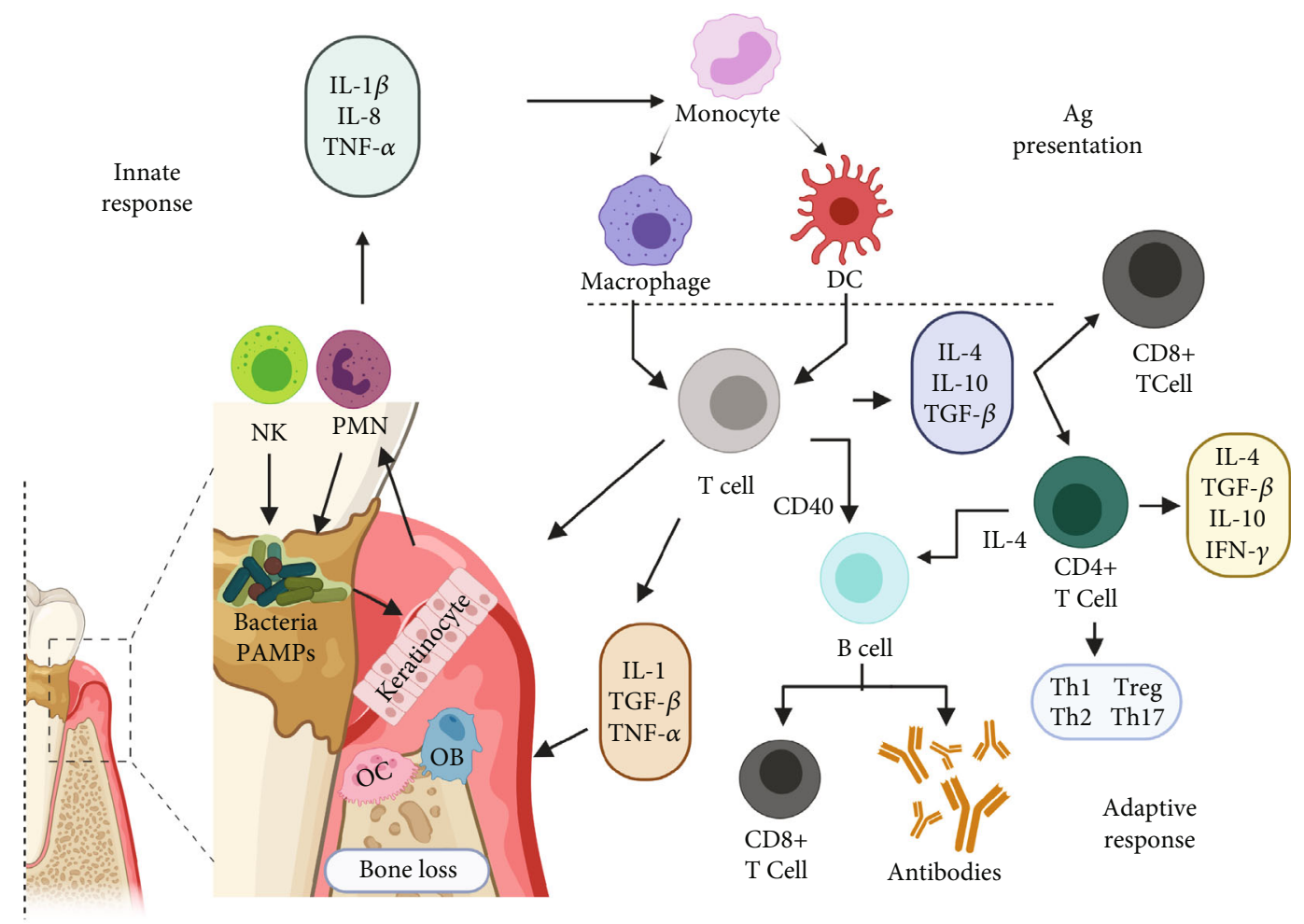

FIGURE 1: Innate immune cells, including keratinocytes, polymorphonuclear cells (PMNs), antigen-presenting cells (APCs), and natural killer (NK) cells, hold pathogen recognition receptors (PRRs) that detect pathogen-associated molecular patterns (PAMPs) present in biofilm bacteria, and these interactions promote the acute inflammatory response. Antigen-presenting cells (APCs) phagocytose pathogens, process antigens, and present the antigens in the form of peptides displayed by major histocompatibility complex (HLA) molecules. Costimulatory molecules stabilize this interaction. APCs migrate to secondary lymphoid tissues where they activate adaptive immune cells, including $\mathrm{CD}^{+} \mathrm{T}$ cells, such as Th1, Th2, and Th17 cells; cytotoxic $\mathrm{CD} 8^{+} \mathrm{T}$ cells; and B cells that will mature into antibody-producing plasma cells. Created with BioRender.com (https://biorender.com/).

Foxp $3^{+} \mathrm{T}$ (Treg) cells [38], which also regulate the inflammatory Th1 response, thus, preventing the destruction of periodontal tissues [39].

Th17 cells are characterized by the production of the cytokine IL-17, although they also produce other cytokines, such as IL-17F, IL-21, IL-22, and GM-CSF [40]. Th17 cells are induced in the presence of TGF- $\beta /$ IL- $1 \beta$, IL- 6 , and IL23 and express chemokine receptor- (CCR-) 6 , which allows their migration to barrier and mucosal sites, such as gingival tissues, suggesting a protective role in the oral barrier. The inflammatory functions of Th17 cells depend on the different combinations of cytokines expressed in the local environment [33].

IL-17 signalling on epithelial cells is essential for the physiological regulation of mucosal immunity and barrier defences, promotes the production of antimicrobial factors, regulates the recruitment and generation of neutrophils through the induction of chemokines CXCL-1, 2, and 5, and induces the secretion of granulopoietic factors such as G-CSF and GM-CSF. Moreover, IL-17 induces the production of antimicrobial mediators such as $\beta$-defensins (HBD), regenerative proteins $(\mathrm{ReG}), \mathrm{S} 100$ proteins, cathelicidins, lipocalins, and lactoferrins [40].

In periodontal lesions, an increase in Th17-related cytokines, such as IL-23 and IL-21, and other proinflammatory and osteoclastogenic mediators, such as IL- 6 and RANKL, has been found [41]. IL-17 can enhance RANKL expression on osteoblasts by promoting the secretion of MMP-1, MMP3 , IL-6, and IL- 8 from gingival fibroblasts and TNF release from macrophages in periodontal tissues [42] and activate RANK signalling on osteoclasts, promoting osteoclastogenesis [43]. In addition, IL-17 enhances inflammation through excessive neutrophil recruitment, enhances proinflammatory cytokine production, and activates osteoclasts, contributing to immunopathology and bone destruction.

In addition, the presence of Treg cells inhibits osteoclast formation and monocyte $/ \mathrm{M} \varphi$ differentiation through the secretion of transforming growth factor- (TGF-) $\beta$, IL-4, and IL-10 and the interaction of CTLA-4 (cytotoxic Tlymphocyte antigen) with the monocyte precursor/M $\varphi$ receptors CD80/CD86 [44].

Keratinocytes express several families of pattern recognition receptors, including TLR2, TLR4, NOD1, and NOD2, which are activated by both extracellular and intracellular bacterial molecular structures.

The mechanisms of tolerance include not only DCs but also Tregs. The function of oral Langerhans cells (LCs) under physiological conditions is to maintain a state of immune tolerance [45]. DCs also participate in peripheral tolerance in chronic periodontitis. These cells are capable of phagocytosing pathogens, but due to the antiinflammatory cytokines IL-10 and TGF- $\beta$, their ability to 
present antigens decreases; this decrease is associated with a deficiency in the costimulatory molecules CD80 and CD86, so they cannot activate T cells properly [29]. Natural killer cells, either through direct cell-to-cell contact or indirectly through cytokines, interact with dendritic cells to mediate $\mathrm{T}$ cell immune responses [46].

In summary, the severity of the pathology of periodontal disease, as well as its chronicity, depends on the balance and interaction between the Th1/Th17 inflammatory response and the Th2/Treg anti-inflammatory regulatory response [47]. The Treg/Th17 balance is shifted in favour of Th17 cells in the presence of proinflammatory cytokines.

\section{Remodelling of Bone Tissue in Periodontitis}

Bone tissue is one of the most affected tissues in periodontitis; under normal conditions, it is constantly remodelled, which requires cells that degrade the bone matrix (osteoclasts) and cells that synthesize the bone matrix (osteoblasts) [48]. Briefly, the bone matrix produces the growth factors TGF- $\beta$ and insulin-like growth factor- (IGF-) 1 . Both molecules favour the recruitment of preosteoblasts and promote their maturation; subsequently, some osteoblasts differentiate into osteocytes. This mechanism is regulated by paracrine and endocrine factors, such as epinephrine B2, IL-6, and parathyroid hormone (PTH) [49].

On the other hand, osteoclasts differentiate from a myeloid precursor under the influence of $\mathrm{M} \varphi$-colony-stimulating growth factor (M-CSF) and RANKL. Osteoprotegerin (OPG), produced by osteoblasts, modulates the osteoclast differentiation process [50]. Osteoclasts produce the proteolytic enzymes cathepsin K and metalloproteinases (MMPs), which degrade the bone matrix. In addition, $\mathrm{H}^{+}$proton transporters and ATPase generate an acidic environment that, together with chloride channels, hydrolyses, and solubilizes both organic matter and inorganic matter. All this happens in Howship's lacunae, and the osteoclasts seal them with their podosomes [51].

In periodontitis, lymphocyte infiltrates and mononuclear cells influence and alter the homeostatic balance of the bone. Although modulation of the bone immune system is complex, the balance of proinflammatory Th1 and antiinflammatory Th2 immune responses is critical [52]. The cytokines that promote bone resorption include IL- $1 \beta$, TNF- $\alpha$, IL-6, IL-15, and IL-17, and the cytokines that inhibit bone resorption include IL-4, IL-10, IL-13, IL-18, GMCSF, and IFN- $\gamma$ [50]. The best example is TNF- $\alpha$, which activates osteoclasts and inhibits osteoblast differentiation with a consequent decrease in bone formation [53]. Specifically, TRAIL (TNF-related apoptosis-inducing ligand) participates in osteoblast apoptosis and low bone quality in periodontitis [54].

The other important factor in bone resorption is a member of the TNF family, RANKL, which promotes osteoclast differentiation and modifies the relationship between osteoblasts and osteoclasts [55]. RANKL is overexpressed in proinflammatory systems, and the major source is B lymphocytes, followed by $\mathrm{T}$ lymphocytes and finally monocytes, although osteoblasts also produce RANKL after activation through TLRs [56].

The RANKL-RANK-OPG system is involved in bone regulation via regulation of the immune system to control other systems and several pathologies. These interactions have been described mainly in rheumatoid arthritis, which involves bone loss and bone remodelling [57, 58]. The regulation of the RANKL-RANK-OPG system and its mechanisms should be clarified in periodontitis since modulation of these mechanisms may favour treatment and prevent disease sequelae.

4.1. Involvement of Metalloproteinases in Bone Remodelling. Periodontal tissues are composed of connective tissue; the extracellular matrix (ECM) is mainly formed by collagen types I, III, IV, V, and VI and noncollagenous proteins, including elastin, fibronectin, laminins, and proteoglycans. In periodontitis, significant degradation of all of these constituent elements of periodontal tissues occurs. Overexpression of MMPs, a family of zinc-dependent endopeptidases, is associated with the development and severity of periodontal structure loss in this pathology. These enzymes are capable of degrading most of the components of the ECM [59]. In addition, MMPs favour processes involved in inflammation, such as inflammatory cell migration, chemokine recruitment and processing, cleavage and neutralization of complement components, phagocytosis, and cell lysis [60].

Different MMPs have the specificity to act in the degradation of specific types of tissues, e.g., MMP-2 in its proenzyme form $(\sim 72 \mathrm{kDa})$ and in its active form $(\sim 59-62 \mathrm{kDa})$ and the MMP-9 proenzyme $(\sim 92 \mathrm{kDa})$ and active forms $(\sim 88 \mathrm{kDa})$ degrade fibronectin, elastin, and collagen types IV, V, VII, X, XI, and XII; in acidic medium, they can degrade collagen type I [61]. MMP-13, both in its proenzyme form $(\sim 60 \mathrm{kDa})$ and in its active form $(\sim 45-50 \mathrm{kDa})$, degrades collagen I, collagen II, collagen III, collagen IV basal membrane, proteoglycans, fibronectin, fibrin, and tenasin [62].

The mechanisms that regulate homeostasis, such as the overexpression of MMPs in periodontitis, are complex processes. In homeostasis, one of the regulatory pathways involves tissue inhibitors of MMPs (TIMPS) and $\alpha 2$-macroglobulins that bind covalently and irreversibly to the active site of MMPs with high affinity. The levels of these endogenous mediators are elevated in healthy tissue and various fluids, such as serum, amniotic fluid, and saliva, and these mediators are synthesized by fibroblasts, monocytes, $\mathrm{M} \varphi \mathrm{s}$, endothelial cells, and osteoblasts [63]. Another mechanism of negative regulation of MMP expression is the presence of the oestrogen $17 \beta$-oestradiol, which negatively regulates the flow of calcium into cells [64] and consequently reduces the expression of MMPs, particularly MMP-1 [65].

On the other hand, the overexpression of MMPs can be triggered by different factors, such as the presence of $\mathrm{PGE}_{2}$ [66], in vitro and in vivo are influenced by mechanical load as orthodontic movement $[67,68]$, interactions with periodontopathogenic bacteria, such as Eikenella corrodens [69], Porphyromonas gingivalis, and Prevotella intermedia [70], or polysaccharides and cytokines, such as IL- $1 \beta$ and TNF- 
TABLE 1: Influence of sex hormones on some cells and cytokines of the immune response.

\begin{tabular}{|c|c|c|}
\hline Hormone & Regulation & References \\
\hline $17 \beta$-estradiol & $\begin{array}{c}\uparrow \text { TCD8+ from spleen and in vitro. } \\
\uparrow \text { maturation and activation of B lymphocytes, } \downarrow \text { Ig } 2 \mathrm{a} \text { in peripheral blood mononuclear cells and spleen cells. } \\
\downarrow \text { TNF- } \alpha \text {, } \uparrow \text { IFN- } \gamma \text { e } \uparrow \text { IL- } 10 \text { in peripheral blood mononuclear cells, spleen, and in vitro. } \\
\text { Peritoneal M } \varphi \uparrow \text {, TLR4 in vitro. } \\
\uparrow \text { DCs } \uparrow \text { IL- } 12 \text { in bone marrow, in vivo. } \\
\text { Inhibits apoptosis by TNF- } \alpha \text { via PI3k/Akt in neural progenitor cells. } \\
\downarrow \text { IL- } 1 \beta \text { and TNF- } \alpha \text { in bone marrow. } \\
{[\downarrow \mathrm{E} 2] \uparrow T h 1,[\uparrow E 2] \uparrow T h 2 \text { in peripheral blood mononuclear cells in vitro. }}\end{array}$ & $\begin{array}{l}{[75]} \\
{[76,77]} \\
{[78,79]} \\
{[80]} \\
{[81]} \\
{[82]} \\
{[83]} \\
{[84]}\end{array}$ \\
\hline Progesterone & $\begin{array}{c}\downarrow \mathrm{M} \varphi \text {, DCs, and NKs in peripheral blood mononuclear cells } \\
\downarrow \text { NF } \kappa \text { B transduction. } \\
\uparrow \mathrm{Th} 2, \uparrow \mathrm{IL}-4 \text { e } \uparrow \mathrm{IL}-5, \uparrow \text { Tregs, and } \downarrow \text { TH17 in peripheral blood mononuclear cells. }\end{array}$ & $\begin{array}{c}{[85]} \\
{[86]} \\
{[87,88]}\end{array}$ \\
\hline Testosterona & $\begin{array}{c}\downarrow \mathrm{LB}, \uparrow \text { apoptosis in bone marrow and lymph nodes. } \\
\uparrow \mathrm{TCD} 8+\text { in peripheral blood mononuclear cells. } \\
\uparrow \mathrm{M} \varphi, \uparrow \mathrm{TNF}-\alpha, \uparrow \mathrm{CCR} 2, \uparrow[\text { IL-10], and } \downarrow \text { IFN- } \gamma \text { in skin and spleen. } \\
\text { M } \varphi, \uparrow \text { IL-12 e } \uparrow \text { IL- } 1 \beta \text { in vitro. } \\
\text { DCs } \downarrow \text { TNF- } \alpha \text {, nitric oxide, TLR- } 4 \text {. } \\
\downarrow \text { IgG e IgM, peripheral blood mononuclear cells. } \\
\uparrow \mathrm{TGF}-\beta \text { e } \uparrow \text { IGFs } \uparrow \text { bone apposition, } \downarrow \text { IL- } 6 \text { osteoclastogenesis. }\end{array}$ & $\begin{array}{c}{[89]} \\
{[90]} \\
{[91,92]} \\
{[93]} \\
{[94,95]} \\
{[96]} \\
{[97,98]}\end{array}$ \\
\hline
\end{tabular}

$\alpha$. In any case, these factors act on monocytes and $\mathrm{M} \varphi$ s by favouring the production of mediators that function as activators or modulators of MMPs [71]. For example, MMP-13 upregulates RANKL/OPG levels by activating MMP-9, increases TGF- $\beta$ signalling in metastatic bone lesions [72], and influences osteoclastic activity [73].

Undoubtedly, the participation of MMPs in the development and severity of periodontitis is known. Establishing whether their expression is affected by hormonal conditions, such as gestation, is important because regulating their expression could be considered a component of the therapeutic treatment of gestational periodontitis.

\section{Sex Hormones and Periodontitis}

Sex hormones modulate immune functions, such as thymocyte maturation and selection, cell migration, MHC-II expression, cell proliferation, and cytokine production (Table 1) [74].

The regulatory effects exerted by hormones on the immune response depend on interactions with their receptors. For example, B cells have high expression of the genes encoding the two oestrogen receptors, ER1 and ER2. There is a moderate expression of these receptors on $\mathrm{CD}^{+} \mathrm{T}$, $\mathrm{CD}^{+} \mathrm{T}, \mathrm{NK}$, and plasmacytoid DCs, while monocytes express reduced levels of ER1. Estradiol and ERs bind to transcription factors, such as $\mathrm{NF} \kappa \mathrm{B}, \mathrm{SP} 1, \mathrm{AP}-1$, and $\mathrm{C} / \mathrm{EBP} \beta$, that are involved in the regulation of different cellular functions [99].

Progesterone receptors (PRs) are present on epithelial cells, mast cells, eosinophils, NK cells, M $\varphi s$, plasmacytoid DCs, and $\mathrm{CD}^{+}$and $\mathrm{CD}^{+} \mathrm{T}$ lymphocytes. Interestingly, the expression of PRs is higher in DCs from female rats than in those from male rats [100], which makes it clear that the expression of these receptors and consequently the response that is generated when their ligand binds are higher in females. Different PRs include two intracellular receptors (iPRs) and three membrane receptors (mPRs), with two isoforms each. iPRs were initially described in the lymphocytes of pregnant women, while mPRs were described in T lymphocytes and are overexpressed during the luteal phase in $\mathrm{CD}^{+} \mathrm{T}$ lymphocytes. Differential expression of PRs may partially explain the differential activation of immune cells and differences in susceptibility to various infectious and noninfectious diseases between men and women [101].

In the same context, the sex hormone profile also has an impact on subgingival microbiology. It has been demonstrated that this profile promotes the development of periodontopathogenic bacteria, such as Porphyromonas gingivalis [102], subgingival anaerobic-aerobic bacteria, Prevotella melaninogenica, and Prevotella intermedia [103]. It is widely recognized that hormones related to gestation alter the immune response, modifying the pathogenesis of some diseases; for example, in multiple sclerosis and autoimmune encephalomyelitis, where an exacerbated inflammatory response is associated with the severity of the pathology, the disease severity decreases during gestation. Diseases such as malaria and influenza, which require acute inflammatory responses for their control, are exacerbated during pregnancy [80]. This phenomenon could be associated with estriol concentrations, which increase significantly during gestation. Estriol is produced in high concentrations by the fetoplacental unit during pregnancy; it accounts for $90 \%$ of the oestrogen produced during pregnancy, while the other $10 \%$ corresponds to oestradiol [104]. Although the immunological functions of estriol are similar to those of oestradiol because they share receptors, estriol seems to differentially influence the immune response; in experimental models of autoimmune pathologies, when estriol was administered, decreases in the proinflammatory cytokines TNF- $\alpha$ and IFN- $\gamma$ have been observed, in addition to decreases in $\mathrm{CD}^{+}$and $\mathrm{CD}^{+}$cells [105]. This immune response modified 
by the presence of estriol, together with other hormones present during pregnancy, could also influence the development of periodontitis. This observation is corroborated in pregnant women, who, due to their condition, have a modified hormonal profile that consequently favours the accumulation of Bacteroides, which is increased in abundance up to 55 times in pregnant women compared to nonpregnant women [106].

The involvement of hormones other than estriol in the development of periodontal diseases has been widely documented. Progesterone increases vascular permeability and favours oedema, erythema, and gingival bleeding, which are all associated with increased populations of Porphyromonas gingivalis, Prevotella intermedia [107], Actinobacillus actinomycetemcomitans [108], and Prevotella melaninogenica [109].

Oestrogens, particularly oestradiol, favour angiogenesis and fibroblast proliferation and promote osteoblast differentiation and maturation, osteoprotegerin (OPG) and RANKL expression in osteoblasts, and osteoclast apoptosis by inhibiting osteoclast activity [83]. Periodontal ligament (PLD) cells synthesize RANKL and OPG. In vitro cultures of oestrogen-treated PLD cells increase OPG expression and decrease RANKL expression through ER2 [110]; these observations demonstrate that oestrogens can modulate the activity of periodontal tissues and promote homeostasis.

Androgens participate in bone growth; they are anabolic agents that increase bone mass, mainly in males, although different androgens, including testosterone, are also present in females. Androgen receptor mRNA is expressed more in cortical osteoblasts than in trabecular bone and is more closely related to cortical osteoblasts, which generate a thicker cortical bone layer in males, while in osteoblasts, androgen receptor mRNA is expressed similarly between the sexes. Androgens promote osteoblast differentiation and decrease osteoclast apoptosis; specifically, dihydrotestosterone reduces OPG levels. The functions of androgens in women have not been clearly defined; however, they are involved in the maintenance of bone density [100].

Sex hormones are involved in bone regulation and immune system maturation and modulate the function of nonsexual tissues; therefore, these hormones may play a central role in the development of periodontitis in different stages of life.

5.1. Pregnancy and Periodontitis. Gestation is a condition that involves physiological changes in the mother, and these changes should allow "immune tolerance" towards the foetus to develop, as well as the appearance of new cells, such as trophoblasts [111].

Recently, the relevance of the model of a foetus as a semiallograft capable of inducing the absence of a specific immune response to prevent its destruction has been debated. It is not a simple absence of the immune response but a state of immunoregulation that allows the implantation of a foetus, which is also able to respond to injury or aggression from the environment with an immune response endowed with specificity and memory [112]. This immune tolerance, in order to not reject the foetus and at the same time allow protection of pregnant woman against pathogens, requires the transient modification of immunity, which favours a Th2 environment over a Th1 environment [113]. Different mechanisms have been described to explain immune tolerance to paternal antigens, including tolerance induction in T lymphocytes, including Treg and Th17 cells [114]. In a healthy pregnancy, the Th17/Treg ratio shifts in favour of Treg cells, while a decrease in Treg cells or an increase in Th17 cells is detrimental to normal pregnancy [115]. Tolerance is promoted by Treg and Th2 cells by repressing Th1 and Th17 cells, while Th17 cells protect trophoblasts from pathogens [116].

During gestation, the maternal-foetal interaction and the development of the placenta favour increased hormone concentrations. In particular, the placenta synthesizes and releases oestrogen and progesterone into circulation. This initiates events that stimulate "suppressive" immune responses, mainly at the level of lymphocytes. Suppression of $\mathrm{CD}^{+}$and $\mathrm{CD} 8^{+} \mathrm{T}$ lymphocytes decreases the secretion of IL-2, IFN- $\gamma$, TNF- $\beta$, TNF- $\alpha$, IL- $1 \beta$, and IL-6 [117]. The levels of oestradiol in human serum are $\sim 0.1 \mu \mathrm{M}$, and those in blood from the intervillous space are $\sim 0.25 \mu \mathrm{M}$, which is $\sim 25$ times higher than the concentration found in nonpregnant women at the midovarian cycle stage [118]. Oestradiol at a concentration of $0.04 \mathrm{ng} / \mathrm{mL}$ or higher and progesterone at $0.1 \mathrm{ng} / \mathrm{mL}$ both inhibit lipopolysaccharide- (LPS-) induced IL- 1 and TNF- $\alpha$ secretion in monocyte cultures. In addition, the switch from the Th1 profile to the Th2 profile and the suppression of the cytolytic function of NK cells are processes regulated by progesterone-induced blocking factor (PIBF), which is secreted by $\mathrm{CD}^{+} \gamma \delta \mathrm{T}$ cells [119]. High concentrations of PIBF favour the differentiation of $\mathrm{CD}^{+}{ }^{+} \mathrm{T}$ cells into Th2 lymphocytes, which increases IL-4, IL-5, and IL-10 concentrations and promotes the prevalence of an anti-inflammatory profile (Figure 2) [120].

In the gestational stage, polymorphonuclear cells (PMNs) show decreased chemotaxis and adhesion beginning in the second trimester and continuing throughout gestation [121]. This altered neutrophil activation and depressed leukocyte function during pregnancy may explain susceptibility to certain infections [122]. For example, gingival inflammation has been associated with increased serum levels of oestrogen and progesterone, even though no changes in TNF- $\alpha$ or IL-1 $\beta$ levels have been detected [123].

In animal models, levels of the cytokines IL- $1 \beta$, IL-6, IL8 , IL-17, and TNF- $\alpha$ increase under different conditions. For example, in maternal infections with periodontal pathogens or in vitro models of placental cells and tissues, exposure to periodontal bacteria or products induces the secretion of COX-2, IL-8, IFN- $\gamma$, and TNF- $\alpha$ in addition to causing apoptosis [124], and $\mathrm{PGE}_{2}$ causes uterine contractions [125].

On the other hand, during pregnancy, periodontal alterations increase, and the ratio of anaerobic to aerobic bacteria is modified in the second trimester of pregnancy, mainly through increases in Prevotella melaninogenica and Prevotella intermedia related to the plasma concentrations of estrogens and progesterone [126]. In animal models of periodontitis established with Porphyromonas gingivalis in pregnant mice, an increased immune response with decreased expression of anti-inflammatory cytokines and increased 


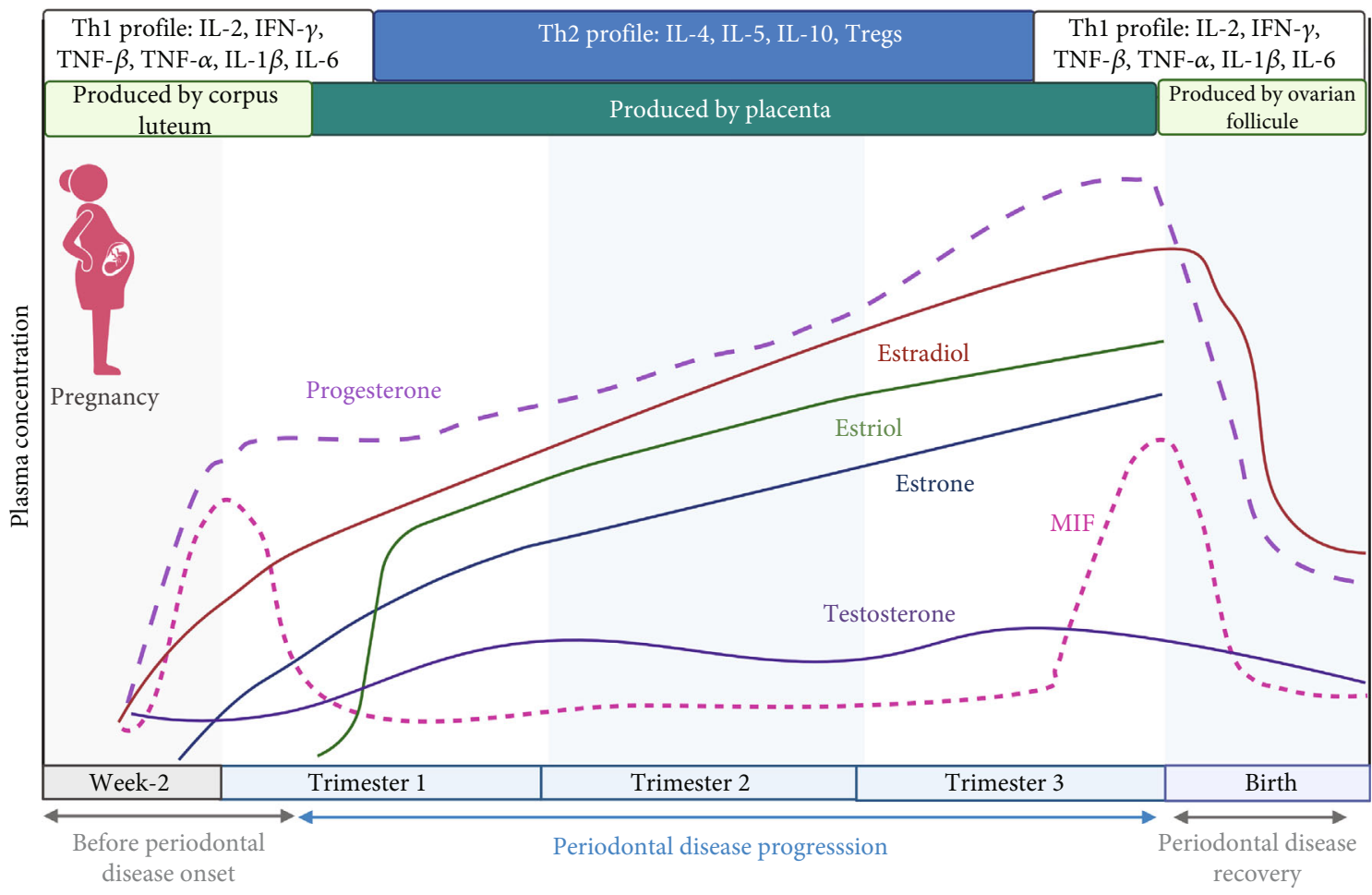

FIGURE 2: Time course of pregnancy hormones, periodontal disease, and the proinflammatory cytokine MIF. During early gestation, hormones are produced in the corpus luteum, and later until the end of gestation, they are produced in the placenta. Estriol is also synthesized in the placenta, and its production ceases when the pregnancy reaches term. Therefore, embryo implantation and early gestation require a Th1 profile with the expression of proinflammatory cytokines. During the second half of gestation, the expression of these cytokines decreases, and the profile is a Th2 and Treg cell profile, which is maintained until delivery, when the profile returns to a Th1 profile. The expression of MIF, which is a proinflammatory cytokine, maintains this trend during pregnancy. Created with BioRender.com (https://biorender.com/).

destruction of periodontal tissues is observed [102], and an imbalance in the Th17/Treg cell ratio with aggravation of periodontitis during pregnancy occurs [127].

Periodontitis causes gynecological problems, ranging from difficulty in embryo implantation to preterm delivery and low birth weight. Two possible causes have been proposed: first, periodontal bacteria cause infections in the placenta and foetus; second, inflammation can provoke responses at the maternal-foetal interface [128]. There are several contrasting and inconclusive reports on patients with recurrent miscarriages and multiple implantation failures during in vitro fertilization cycles which have a prevalent Th1 profile in their peripheral blood lymphocytes [129].

Offenbacher et al. noted that primary infections in distant systems can guide a pregnancy to an abnormal term [130]. Periodontal disease is an infectious process in periodontal tissues characterized by an increase in proinflammatory cytokines, and during pregnancy, the concentration of prostaglandins increases [35]. Therefore, there may be a relationship between both factors; periodontitis influences pregnancy, and that gestation influences the severity of periodontitis. One of the possible causes is the spread of bacteria or inflammatory mediators of periodontal origin by different routes, including (1) bacterial blood spread (bacteremia), (2) blood dissemination of inflammatory mediators, and (3) transmission of oral pathogens and colonization of the vag- inal microbiome [131]. González-Jaranay et al. reported that in pregnant women with some degree of periodontitis, symptoms progress and worsen throughout gestation. However, in the postpartum period, clinical data improve [132]. Other authors have noted that maternal periodontal disease is not a risk factor if infectious processes are controlled [133].

Regarding the interaction of gestation with periodontitis, some studies did not find strong evidence of this interaction; however, they proposed routine periodontal therapy in pregnant women as a safe treatment for mothers and foetuses, in addition to improving the clinical signs of maternal periodontal disease [134]. In contrast, evidence of strong links between periodontitis and pregnancy disorders such as preeclampsia, preterm delivery, and low birth weight, attributable to periodontal disease, has recently been reported [135], as have associations of periodontitis with metabolic disorders such as obesity and diabetes [136].

\section{Conclusions}

This review shows that sex hormones modulate the immune response and participate in processes such as the maturation and selection of immune cells, cell trafficking, expression of histocompatibility molecules, cell proliferation, and cytokine production. Although pregnancy is a condition that modifies 
the hormonal profile, little is known about its effects on the development of periodontitis. Here, we collect important evidence that gestational hormones, such as $17 \beta$ oestradiol, estriol, and progesterone, influence the development of periodontitis. Importantly, the interaction between the concentration of gestational hormones and periodontal disease appears to be bidirectional: on the one hand, the hormonal profile during pregnancy seems to be decisive for the development and severity of periodontal disease, but on the other hand, the infectious process associated with periodontitis during pregnancy generates a proinflammatory immune profile that can produce alterations such as preeclampsia, preterm delivery, and low birth weight.

However, future studies are needed to understand the immune mechanisms underlying the interaction of pregnancy and periodontal diseases. The information gathered here has the potential to contribute to an understanding of the role of hormones in the development of periodontitis, allowing dental teams that care for pregnant and childbearing women to develop preventive and therapeutic strategies.

\section{Data Availability}

No data were used to support this study.

\section{Conflicts of Interest}

The authors declare that there is no conflict of interest regarding the publication of this paper.

\section{Acknowledgments}

Betsaida Julieta Ortiz-Sánchez is a doctoral student from the Programa de Doctorado en Ciencias Biológicas, Universidad Nacional Autónoma de México (UNAM), and received a fellowship from the Council for Science and Technology of the State of Mexico (COMECyT; 2019-2020). This work was funded in part by the Support Program for Research Projects and Technological Innovation (PAPIIT)-UNAM, grant number (IN-217021) and by the National Council of Science and Technology of Mexico (CONACYT), grant number (A1-S-10463).

\section{References}

[1] H. C. Slavkin, "Does the mouth put the heart at risk?," Journal of the American Dental Association (1939), vol. 130, no. 1, pp. 109-113, 1999.

[2] M. S. Tonetti, S. Jepsen, L. Jin, and J. Otomo-Corgel, "Impact of the global burden of periodontal diseases on health, nutrition and wellbeing of mankind: a call for global action," Journal of Clinical Periodontology, vol. 44, no. 5, pp. 456-462, 2017.

[3] A. Duque, "Chronic periodontitis in Latin America," Revista clínica de periodoncia, implantología y rehabilitación oral, vol. 9, no. 2, pp. 208-215, 2016.

[4] L. Ramaglia, G. Isola, G. Matarese et al., "Prophylaxis of acute attacks with a novel short-term protocol in hereditary angioedema patients requiring periodontal treatment," Oral Health \& Preventive Dentistry, vol. 18, no. 1, pp. 355-361, 2020.
[5] C. Verrusio, V. Iorio-Siciliano, A. Blasi, S. Leuci, D. Adamo, and M. Nicolò, Eds., "The effect of orthodontic treatment on periodontal tissue inflammation: a systematic review," Quintessence International, vol. 49, no. 1, pp. 69-77, 2018.

[6] Y. C. Liu, U. H. Lerner, and Y. T. Teng, "Cytokine responses against periodontal infection: protective and destructive roles," Periodontology 2000, vol. 52, no. 1, pp. 163-206, 2010.

[7] R. J. Genco, "Current view of risk factors for periodontal diseases," Journal of Periodontology, vol. 67, no. 10, pp. 10411049, 1996.

[8] C. O. Enwonwu and N. Salako, "The periodontal diseasesystemic health-infectious disease axis in developing countries," Periodontology 2000, vol. 60, no. 1, pp. 64-77, 2012.

[9] G. Isola, G. Matarese, L. Ramaglia, E. Pedullà, E. Rapisarda, and V. Iorio-Siciliano, "Association between periodontitis and glycosylated haemoglobin before diabetes onset: a cross-sectional study," Clinical Oral Investigations, vol. 24, no. 8, pp. 2799-2808, 2020.

[10] G. Isola, R. C. Williams, A. Lo Gullo et al., "Risk association between scleroderma disease characteristics, periodontitis, and tooth loss," Clinical Rheumatology, vol. 36, no. 12, pp. 2733-2741, 2017.

[11] D. A. Webb, L. Mathew, and J. F. Culhane, "Lessons learned from the Philadelphia collaborative preterm prevention project: the prevalence of risk factors and program participation rates among women in the intervention group," BMC Pregnancy and Childbirth, vol. 14, no. 1, p. 368, 2014.

[12] B. L. Mealey and A. J. Moritz, "Hormonal influences: effects of diabetes mellitus and endogenous female sex steroid hormones on the periodontium," Periodontology 2000, vol. 32, no. 1, pp. 59-81, 2003.

[13] Y. Shimaoka, Y. Hidaka, H. Tada et al., "Changes in cytokine production during and after normal pregnancy," American Journal of Reproductive Immunology, vol. 44, no. 3, pp. 143-147, 2000.

[14] A. D. Proal, I. A. Lindseth, and T. G. Marshall, "Microbemicrobe and host-microbe interactions drive microbiome dysbiosis and inflammatory processes," Discovery Medicine, vol. 23, no. 124, pp. 51-60, 2017.

[15] A. Radaic and Y. L. Kapila, "The oralome and its dysbiosis: new insights into oral microbiome-host interactions," Computational and Structural Biotechnology Journal, vol. 19, pp. 1335-1360, 2021.

[16] R. J. Lamont, H. Koo, and G. Hajishengallis, "The oral microbiota: dynamic communities and host interactions," Nature Reviews Microbiology, vol. 16, no. 12, pp. 745-759, 2018.

[17] L. Tonoyan, S. Vincent-Bugnas, C. V. Olivieri, and A. Doglio, "New Viral Facets in Oral Diseases: The EBV Paradox," International Journal of Molecular Sciences, vol. 20, no. 23, p. 5861, 2019.

[18] B. J. Paster, S. K. Boches, J. L. Galvin et al., "Bacterial diversity in human subgingival plaque," Journal of Bacteriology, vol. 183, no. 12, pp. 3770-3783, 2001.

[19] H. T. Huynh, M. Pignoly, V. D. Nkamga, M. Drancourt, and G. Aboudharam, "The repertoire of archaea cultivated from severe periodontitis," PLoS One, vol. 10, no. 4, article e0121565, 2015.

[20] C. J. Nobile and A. D. Johnson, "Candida albicansBiofilms and human disease," Annual Review of Microbiology, vol. 69, no. 1, pp. 71-92, 2015. 
[21] J. Slots, "Periodontal herpesviruses: prevalence, pathogenicity, systemic risk," Periodontol 2000, vol. 69, no. 1, pp. 2845, 2015.

[22] F. E. Dewhirst, T. Chen, J. Izard et al., "The human oral microbiome," Journal of Bacteriology, vol. 192, no. 19, pp. 5002-5017, 2010.

[23] H. Y. Kim, Y. Lim, S. J. An, and B. K. Choi, "Characterization and immunostimulatory activity of extracellular vesicles fromFilifactor alocis," Molecular Oral Microbiology, vol. 35, no. 1, pp. 1-9, 2020.

[24] A. W. Aruni, K. Zhang, Y. Dou, and H. Fletcher, "Proteome analysis of coinfection of epithelial cells with Filifactor alocis and Porphyromonas gingivalis shows modulation of pathogen and host regulatory pathways," Infection and Immunity, vol. 82, no. 8, pp. 3261-3274, 2014.

[25] G. Hajishengallis and R. J. Lamont, "Beyond the red complex and into more complexity: the polymicrobial synergy and dysbiosis (PSD) model of periodontal disease etiology," Molecular Oral Microbiology, vol. 27, no. 6, pp. 409-419, 2012.

[26] Y. Delneste, C. Beauvillain, and P. Jeannin, "Immunité naturelle,” Medical Science (Paris), vol. 23, no. 1, pp. 67-74, 2007.

[27] S. A. Khan, E. F. Kong, T. F. Meiller, and M. A. Jabra-Rizk, "Periodontal diseases: bug Induced, Host Promoted," PLOS Pathogens, vol. 11, no. 7, article e1004952, 2015.

[28] D. F. Kinane, P. G. Stathopoulou, and P. N. Papapanou, "Periodontal diseases," Nature Reviews Disease Primers, vol. 3, no. 1, 2017.

[29] M. Azuma, "Fundamental mechanisms of host immune responses to infection," Journal of Periodontal Research, vol. 41, no. 5, pp. 361-373, 2006.

[30] K. Hirahara and T. Nakayama, "CD4+ T-cell subsets in inflammatory diseases: beyond the Th1/Th2 paradigm," International Immunology, vol. 28, no. 4, pp. 163-171, 2016.

[31] F. Ietta, E. A. V. Ferro, E. Bevilacqua, L. Benincasa, E. Maioli, and L. Paulesu, "Role of the macrophage migration inhibitory factor (MIF) in the survival of first trimester human placenta under induced stress conditions," Scientific Reports, vol. 8, no. $1,2018$.

[32] H. Terheyden, B. Stadlinger, M. Sanz, A. I. Garbe, and J. Meyle, "Inflammatory reaction - communication of cells," Clinical Oral Implants Research, vol. 25, no. 4, pp. 399-407, 2014.

[33] L. Abusleme and N. M. Moutsopoulos, "IL-17: overview and role in oral immunity and microbiome," Oral Diseases, vol. 23, no. 7, pp. 854-865, 2017.

[34] V. P. B. Parachuru, D. E. Coates, T. J. Milne, A. M. Rich, and G. J. Seymour, "FoxP3 ${ }^{+}$regulatory T cells, interleukin 17 and mast cells in chronic inflammatory periodontal disease," Journal of Periodontal Research, vol. 53, no. 4, pp. 622-635, 2018.

[35] K. Yamazaki, T. Nakajima, E. Gemmell, B. Polak, G. J. Seymour, and K. Hara, "IL-4- and IL-6-producing cells in human periodontal disease tissue," Journal of Oral Pathology \& Medicine, vol. 23, no. 8, pp. 347-353, 1994.

[36] Y. Matsuki, T. Yamamoto, and K. Hara, "Detection of inflammatory cytokine messenger RNA (mRNA)-expressing cells in human inflamed gingiva by combined in situ hybridization and immunohistochemistry," Immunology, vol. 76, no. 1, pp. 42-47, 1992.

[37] N. Stein, "Position Paper:Diabetes and periodontal diseases," Journal of Periodontology, vol. 71, no. 4, pp. 664-678, 2000.
[38] C. F. Francisconi, A. E. Vieira, M. C. S. Azevedo et al., "RANKL triggers Treg-mediated immunoregulation in inflammatory osteolysis," Journal of Dental Research, vol. 97, no. 8, pp. 917-927, 2018.

[39] C. Alvarez, C. Rojas, L. Rojas, E. A. Cafferata, G. Monasterio, and R. Vernal, "Regulatory T lymphocytes in periodontitis: a translational view," Mediators of Inflammation, vol. 2018, Article ID 7806912, 10 pages, 2018.

[40] S. C. Liang, X. Y. Tan, D. P. Luxenberg et al., "Interleukin (IL)-22 and IL-17 are coexpressed by Th17 cells and cooperatively enhance expression of antimicrobial peptides," The Journal of Experimental Medicine, vol. 203, no. 10, pp. 2271-2279, 2006.

[41] S. R. Lester, J. L. Bain, R. B. Johnson, and F. G. Serio, "Gingival concentrations of interleukin-23 and -17 at healthy sites and at sites of clinical attachment loss," Journal of Periodontology, vol. 78, no. 8, pp. 1545-1550, 2007.

[42] K. Bunte and T. Beikler, "Th17 cells and the IL-23/IL-17 axis in the pathogenesis of periodontitis and immune-mediated inflammatory diseases," International Journal of Molecular Sciences, vol. 20, no. 14, p. 3394, 2019.

[43] P. Miossec and J. K. Kolls, "Targeting IL-17 and $\mathrm{T}_{\mathrm{H}} 17$ cells in chronic inflammation," Nature Reviews Drug Discovery, vol. 11, no. 10, pp. 763-776, 2012.

[44] M. E. L. Sommer, R. A. Dalia, A. V. B. Nogueira et al., "Immune response mediated by Th1 / IL-17 / caspase-9 promotes evolution of periodontal disease," Archives of Oral Biology, vol. 97, pp. 77-84, 2019.

[45] R. Jotwani and C. W. Cutler, "Multiple dendritic cell (DC) subpopulations in human gingiva and association of mature DCs with CD4+ T-cells in situ," Journal of Dental Research, vol. 82, no. 9, pp. 736-741, 2003.

[46] K. Hoebe, E. Janssen, and B. Beutler, "The interface between innate and adaptive immunity," Nature Immunology, vol. 5, no. 10, pp. 971-974, 2004.

[47] T. Kawai, T. Matsuyama, Y. Hosokawa et al., "B and T lymphocytes are the primary sources of RANKL in the bone resorptive lesion of periodontal disease," The American Journal of Pathology, vol. 169, no. 3, pp. 987-998, 2006.

[48] E. Könönen, M. Gursoy, and U. K. Gursoy, "Periodontitis: a multifaceted disease of tooth-supporting tissues," Journal of Clinical Medicine, vol. 8, no. 8, p. 1135, 2019.

[49] A. Cappariello, A. Maurizi, V. Veeriah, and A. Teti, “_The Great Beauty_ of the osteoclast," Archives of Biochemistry and Biophysics, vol. 558, pp. 70-78, 2014.

[50] M. C. Walsh, N. Kim, Y. Kadono et al., “Osteoimmunology: interplay between the immune system and bone metabolism," Annual Review of Immunology, vol. 24, no. 1, pp. 3363, 2006.

[51] M. A. Karsdal, K. Henriksen, M. G. Sørensen et al., “Acidification of the osteoclastic resorption compartment provides insight into the coupling of bone formation to bone resorption," The American Journal of Pathology, vol. 166, no. 2, pp. 467-476, 2005.

[52] W. F. Rodrigues, M. F. M. Madeira, T. A. da Silva et al., "Low dose of propranolol down-modulates bone resorption by inhibiting inflammation and osteoclast differentiation," British Journal of Pharmacology, vol. 165, no. 7, pp. 2140-2151, 2012.

[53] G. Luo, F. Li, X. Li, Z. G. Wang, and B. Zhang, "TNF- $\alpha$ and RANKL promote osteoclastogenesis by upregulating RANK 
via the NF- $\kappa$ B pathway," Molecular Medicine Reports, vol. 17, no. 5, pp. 6605-6611, 2018.

[54] A. di Benedetto, I. Gigante, S. Colucci, and M. Grano, "Periodontal disease: linking the primary inflammation to bone loss," Clinical \& Developmental Immunology, vol. 2013, article 503754, pp. 1-7, 2013.

[55] Y. T. Teng, H. Nguyen, X. Gao et al., "Functional human Tcell immunity and osteoprotegerin ligand control alveolar bone destruction in periodontal infection," The Journal of Clinical Investigation, vol. 106, no. 6, pp. R59-R67, 2000.

[56] M. C. Walsh and Y. Choi, "Biology of the RANKL-RANKOPG system in immunity, bone, and beyond," Frontiers in Immunology, vol. 5, p. 511, 2014.

[57] Y. Esparza-Guerrero, C. A. Nava-Valdivia, A. M. SaldañaCruz et al., "El sistema RANK/RANKL/OPG y sus implicaciones clínicas en la osteoporosis," El Residente, vol. 11, no. 3, pp. 99-104, 2016.

[58] Q. Zhao, J. Shao, W. Chen, and Y. P. Li, "Osteoclast differentiation and gene regulation," Frontiers in Bioscience, vol. 12, pp. 2519-2529, 2007.

[59] W. Dong, J. Xiang, C. Li, Z. Cao, and Z. Huang, "Increased expression of extracellular matrix metalloproteinase inducer is associated with matrix metalloproteinase- 1 and -2 in gingival tissues from patients with periodontitis," Journal of Periodontal Research, vol. 44, no. 1, pp. 125-132, 2009.

[60] G. S. Butler and C. M. Overall, "Matrix metalloproteinase processing of signaling molecules to regulate inflammation," Periodontology 2000, vol. 63, no. 1, pp. 123-148, 2013.

[61] J. Bartmann, M. Frankenberger, C. Neurohr, O. Eickelberg, E. Noessner, and W. von Wulffen, "A novel role of MMP13 for murine DC function: its inhibition dampens T-cell activation," International Immunology, vol. 28, no. 10, pp. 473-487, 2016.

[62] A. L. Ejeil, S. Igondjo-Tchen, S. Ghomrasseni, B. Pellat, G. Godeau, and B. Gogly, "Expression of matrix metalloproteinases (MMPs) and tissue inhibitors of metalloproteinases (TIMPs) in healthy and diseased human gingiva," Journal of Periodontology, vol. 74, no. 2, pp. 188-195, 2003.

[63] G. Sapna, S. Gokul, and K. Bagri-Manjrekar, "Matrix metalloproteinases and periodontal diseases," Oral Diseases, vol. 20, no. 6, pp. 538-550, 2014

[64] M. Gürsoy, F. Zeidán-Chuliá, E. Könönen et al., "Pregnancyinduced gingivitis and OMICS in dentistry: in silico modeling and In Vivo prospective validation of estradiol-modulated inflammatory biomarkers," OMICS, vol. 18, no. 9, pp. 582590, 2014.

[65] E. Y. Liao and X. H. Luo, "Effects of 17beta-estradiol on the expression of matrix metalloproteinase- $1,-2$ and tissue inhibitor of metalloproteinase-1 in human osteoblast-like cell cultures," Endocrine, vol. 15, no. 3, pp. 291-296, 2001.

[66] M. Nishikawa, Y. Yamaguchi, K. Yoshitake, and Y. Saeki, "Effects of TNFalpha and prostaglandin E2 on the expression of MMPs in human periodontal ligament fibroblasts," Journal of Periodontal Research, vol. 37, no. 3, pp. 167-176, 2002.

[67] C. Behm, M. Nemec, F. Weissinger, M. A. Rausch, O. Andrukhov, and E. Jonke, "MMPs and TIMPs expression levels in the periodontal ligament during orthodontic tooth movement: a systematic review of in vitro and in vivo studies," International Journal of Molecular Sciences, vol. 22, no. 13, p. $6967,2021$.
[68] C. Behm, M. Nemec, A. Blufstein et al., "Interleukin-1 $\beta$ induced matrix metalloproteinase expression in human periodontal ligament-derived mesenchymal stromal cells under in vitro simulated static orthodontic forces," International Journal of Molecular Sciences, vol. 22, no. 3, p. 1027, 2021.

[69] M. Dahan, B. Nawrocki, R. Elkaïm et al., "Expression of matrix metalloproteinases in healthy and diseased human gingiva," Journal of Clinical Periodontology, vol. 28, no. 2, pp. 128-136, 2001.

[70] J. Zhou, J. Zhang, and J. Chao, "Porphyromonas gingivalis promotes monocyte migration by activating MMP-9," Journal of Periodontal Research, vol. 47, no. 2, pp. 236-242, 2012.

[71] M. Solera, R. Jose, M. Conte et al., "Papel de las Metaloproteinasas de la Matriz en la Degradación del Tejido Pulpar: Una revisión literaria," Revista Científica Odontológica, vol. 1, no. 1, pp. 20-26, 2005.

[72] K. C. Nannuru, M. Futakuchi, M. L. Varney, T. M. Vincent, E. G. Marcusson, and R. K. Singh, "Matrix metalloproteinase (MMP)-13 regulates mammary tumor-induced osteolysis by activating MMP9 and transforming growth factor-beta signaling at the tumor-bone interface," Cancer Research, vol. 70, no. 9, pp. 3494-3504, 2010.

[73] N. Silva, L. Abusleme, D. Bravo et al., "Host response mechanisms in periodontal diseases," Journal of Applied Oral Science, vol. 23, no. 3, pp. 329-355, 2015.

[74] R. Hernandez-Bello, K. Nava-Castro, S. Muniz-Hernandez et al., "Beyond the reproductive effect of sex steroids: their role during immunity to helminth parasite infections," Mini Reviews in Medicinal Chemistry, vol. 12, no. 11, pp. 10711080, 2012.

[75] R. Lamason, P. Zhao, R. Rawat et al., "Sexual dimorphism in immune response genes as a function of puberty," $B M C$ Immunology, vol. 7, no. 1, p. 2, 2006.

[76] F. X. LÜ, K. ABEL, Z. MA et al., "The strength of B cell immunity in female rhesus macaques is controlled by CD8+ T cells under the influence of ovarian steroid hormones," Clinical and Experimental Immunology, vol. 128, no. 1, pp. 10-20, 2002.

[77] Y. Fu, L. Li, X. Liu et al., "Estrogen promotes B cell activation in vitro through down-regulating CD80 molecule expression," Gynecological Endocrinology, vol. 27, no. 8, pp. 593596, 2011.

[78] K. Z. Matalka, "The effect of estradiol, but not progesterone, on the production of cytokines in stimulated whole blood, is concentration-dependent," Neuro Endocrinology Letters, vol. 24, no. 3-4, pp. 185-191, 2003.

[79] E. Karpuzoglu-Sahin, B. D. Hissong, and S. Ansar Ahmed, "Interferon-_ $\gamma_{-}$levels are upregulated by 17 _ $\beta_{-}$- estradiol and diethylstilbestrol," Journal of Reproductive Immunology, vol. 52, no. 1-2, pp. 113-127, 2001.

[80] D. P. Robinson and S. L. Klein, "Pregnancy and pregnancyassociated hormones alter immune responses and disease pathogenesis," Hormones and Behavior, vol. 62, no. 3, pp. 263-271, 2012.

[81] M. C. Siracusa, M. G. Overstreet, F. Housseau, A. L. Scott, and S. L. Klein, "17beta-estradiol alters the activity of conventional and IFN-producing killer dendritic cells," Journal of Immunology, vol. 180, no. 3, pp. 1423-1431, 2008.

[82] T. Wang, S. D. Yang, S. Liu, H. Wang, H. Liu, and W. Y. Ding, " $17 \beta$-Estradiol inhibites tumor necrosis Factor- $\alpha$ induced apoptosis of human nucleus pulposus cells via the PI3K/Akt 
pathway," Medical Science Monitor, vol. 22, pp. 4312-4322, 2016.

[83] J. S. Walsh, "Normal bone physiology, remodelling and its hormonal regulation," Surgery, vol. 33, no. 1, pp. 1-6, 2015.

[84] S. L. Klein, "Immune cells have sex and so should journal articles," Endocrinology, vol. 153, no. 6, pp. 2544-2550, 2012.

[85] C. L. Butts, E. Bowers, J. C. Horn et al., "Inhibitory effects of progesterone differ in dendritic cells from female and male rodents," Gender Medicine, vol. 5, no. 4, pp. 434-447, 2008.

[86] L. I. McKay and J. A. Cidlowski, "Molecular control of immune/inflammatory responses: interactions between nuclear factor-kappa B and steroid receptor-signaling pathways," Endocrine Reviews, vol. 20, no. 4, pp. 435-459, 1999.

[87] I. J. Tan, E. Peeva, and G. Zandman-Goddard, "Hormonal modulation of the immune system - A spotlight on the role of progestogens," Autoimmunity Reviews, vol. 14, no. 6, pp. 536-542, 2015.

[88] J. H. Lee, J. P. Lydon, and C. H. Kim, "Progesterone suppresses the mTOR pathway and promotes generation of induced regulatory T cells with increased stability," European Journal of Immunology, vol. 42, no. 10, pp. 2683-2696, 2012.

[89] S. Altuwaijri, K. H. Chuang, K. P. Lai et al., "Susceptibility to autoimmunity and B cell resistance to apoptosis in mice lacking androgen receptor in B cells," Molecular Endocrinology, vol. 23, no. 4, pp. 444-453, 2009.

[90] S. Aboudkhil, A. Zaîd, L. Henry, and J. P. Bureau, "Influence of age, castration, and testosterone on $\mathrm{T}$ cell subsets in healthy and leukemia grafted mice," Biology of the Cell, vol. 95, no. 1, pp. 9-16, 2003.

[91] J. J. Lai, K. P. Lai, K. H. Chuang et al., "Monocyte/macrophage androgen receptor suppresses cutaneous wound healing in mice by enhancing local TNF-alpha expression," The Journal of Clinical Investigation, vol. 119, no. 12, pp. 3739-3751, 2009.

[92] M. K. Angele, M. W. Knöferl, A. Ayala, K. I. Bland, and I. H. Chaudry, "Testosterone and estrogen differently effect Th1 and Th2 cytokine release following trauma-haemorrhage," Cytokine, vol. 16, no. 1, pp. 22-30, 2001.

[93] E. Posma, H. Moes, M. J. Heineman, and M. M. Faas, "The effect of testosterone on cytokine production in the specific and non-specific immune response," American Journal of Reproductive Immunology, vol. 52, no. 4, pp. 237-243, 2004.

[94] S. A. Ahmed, E. Karpuzoglu, and D. Khan, Effects of sex steroids on innate and adaptive immunity, in Sex hormones and immunity to infection, Springer, 2010.

[95] J. J. Corrales, M. Almeida, R. Burgo, M. T. Mories, J. M. Miralles, and A. Orfao, "Androgen-replacement therapy depresses the ex vivo production of inflammatory cytokines by circulating antigen-presenting cells in aging type- 2 diabetic men with partial androgen deficiency," The Journal of Endocrinology, vol. 189, no. 3, pp. 595-604, 2006.

[96] N. Kanda, T. Tsuchida, and K. Tamaki, "Testosterone inhibits immunoglobulin production by human peripheral blood mononuclear cells," Clinical and Experimental Immunology, vol. 106, no. 2, pp. 410-415, 1996.

[97] B. L. Clarke and S. Khosla, "Androgens and bone," Steroids, vol. 74, no. 3, pp. 296-305, 2009.

[98] K. M. Wiren, "Androgens and bone growth: it's location, location, location," Current Opinion in Pharmacology, vol. 5, no. 6, pp. 626-632, 2005.
[99] S. Kovats, "Estrogen receptors regulate innate immune cells and signaling pathways," Cellular Immunology, vol. 294, no. 2, pp. 63-69, 2015.

[100] S. Kovats, E. Carreras, and H. Agrawal, Sex steroid receptors in immune cells, in Sex hormones and immunity to infection, Springer, 2010.

[101] L. A. Cervantes-Candelas, J. Aguilar-Castro, F. O. BuendíaGonzález et al., " $17 \beta$-Estradiol is involved in the sexual dimorphism of the immune response to malaria," Frontiers in Endocrinology, vol. 12, article 643851, 2021.

[102] X. Duan, A. Hays, W. Zhou et al., “_Porphyromonas gingivalis_ induces exacerbated periodontal disease during pregnancy," Microbial Pathogenesis, vol. 124, pp. 145-151, 2018.

[103] C. Ye, Z. Xia, J. Tang et al., "Unculturable and culturable periodontal-related bacteria are associated with periodontal inflammation during pregnancy and with preterm low birth weight delivery," Scientific Reports, vol. 10, no. 1, article 15807, 2020.

[104] D. Tulchinsky, C. J. Hobel, E. Yeager, and J. R. Marshall, "Plasma estrone, estradiol, estriol, progesterone, and 17hydroxyprogesterone in human pregnancy: I. Normal pregnancy," American Journal of Obstetrics and Gynecology, vol. 112, no. 8, pp. 1095-1100, 1972.

[105] S. S. Soldan, A. I. A. Retuerto, N. L. Sicotte, and R. R. Voskuhl, "Immune modulation in multiple sclerosis patients treated with the pregnancy hormone estriol," Journal of Immunology, vol. 171, no. 11, pp. 6267-6274, 2003.

[106] G. M. Knight and A. Bryan Wade, "The effects of hormonal contraceptives on the human periodontium," Journal of Periodontal Research, vol. 9, no. 1, pp. 18-22, 1974.

[107] K. S. Kornman and W. J. Loesche, "The subgingival microbial flora during pregnancy," Journal of Periodontal Research, vol. 15, no. 2, pp. 111-122, 1980.

[108] R. S. S. Massoni, A. M. F. Aranha, F. Z. Matos et al., "Correlation of periodontal and microbiological evaluations, with serum levels of estradiol and progesterone, during different trimesters of gestation," Scientific Reports, vol. 9, no. 1, article 11762, 2019.

[109] P. S. Kumar, "Sex and the subgingival microbiome: do female sex steroids affect periodontal bacteria?," Periodontology 2000, vol. 61, no. 1, pp. 103-124, 2013.

[110] L. Liang, J. F. Yu, Y. Wang, and Y. Ding, "Estrogen regulates expression of osteoprotegerin and RANKL in human periodontal ligament cells through estrogen receptor beta," Journal of Periodontology, vol. 79, no. 9, pp. 1745-1751, 2008.

[111] M. G. Rico-Rosillo and G. B. Vega-Robledo, "Immunological mechanisms involved in pregnancy," Ginecología y Obstetricia de México, vol. 80, no. 5, pp. 332-340, 2012.

[112] G. Mor, P. Aldo, and A. B. Alvero, "The unique immunological and microbial aspects of pregnancy," Nature Reviews Immunology, vol. 17, no. 8, pp. 469-482, 2017.

[113] R. Raghupathy, "Pregnancy: success and failure within the Th1/Th2/Th3 paradigm," Seminars in Immunology, vol. 13, no. 4, pp. 219-227, 2001.

[114] P. Le Bouteiller and A. Bensussan, "Up-and-down immunity of pregnancy in humans," F1000Research, vol. 6, p. 1216, 2017.

[115] J. Qian, N. Zhang, J. Lin et al., “Distinct pattern of Th17/Treg cells in pregnant women with a history of unexplained recurrent spontaneous abortion," Bioscience Trends, vol. 12, no. 2, pp. 157-167, 2018. 
[116] W. Wang, N. Sung, A. Gilman-Sachs, and J. Kwak-Kim, “T helper (Th) cell profiles in pregnancy and recurrent pregnancy losses: Th1/Th2/Th9/Th17/Th22/Tfh cells," Frontiers in Immunology, vol. 11, p. 2025, 2020.

[117] M. Morishita, M. Miyagi, and Y. Iwamoto, "Effects of sex hormones on production of interleukin-1 by human peripheral monocytes," Journal of Periodontology, vol. 70, no. 7, pp. 757-760, 1999.

[118] V. Brazão, C. C. Kuehn, C. D. . Santos, C. M. B. . Costa, J. C. . P. Júnior, and A. A. Carraro-Abrahão, "Endocrine and immune system interactions during pregnancy," Immunobiology, vol. 220, no. 1, pp. 42-47, 2015.

[119] S. M. Blois, R. Joachim, J. Kandil et al., "Depletion of CD8+ cells abolishes the pregnancy protective effect of progesterone substitution with dydrogesterone in mice by altering the Th1/Th2 cytokine profile," Journal of Immunology, vol. 172, no. 10, pp. 5893-5899, 2004.

[120] A. Mariotti and M. Mawhinney, "Endocrinology of sex steroid hormones and cell dynamics in the periodontium," Periodontology 2000, vol. 61, no. 1, pp. 69-88, 2013.

[121] N. Urteaga Mamani, W. Challapa Licidio, and J. Cortez, "Inmunología de la Implantación," Revista SCientifica, vol. 4, p. 48, 2006.

[122] F. Q. Bui, C. L. C. Almeida-da-Silva, B. Huynh et al., "Association between periodontal pathogens and systemic disease," Biomedical Journal, vol. 42, no. 1, pp. 27-35, 2019.

[123] M. Wu, S. W. Chen, W. L. Su et al., "Sex Hormones Enhance Gingival Inflammation without Affecting IL- $1 \beta$ and TNF- $\alpha$ in Periodontally Healthy Women during Pregnancy," Mediators of Inflammation, vol. 2016, Article ID 4897890, 6 pages, 2016.

[124] H. Ren and M. Du, "Role of maternal periodontitis in preterm birth," Frontiers in Immunology, vol. 8, p. 139, 2017.

[125] S. Komine-Aizawa, S. Aizawa, and S. Hayakawa, "Periodontal diseases and adverse pregnancy outcomes," The Journal of Obstetrics and Gynaecology Research, vol. 45, no. 1, pp. 512, 2019.

[126] C. Silva de Araujo Figueiredo, C. Gonçalves Carvalho Rosalem, A. L. Costa Cantanhede, É. B. Abreu Fonseca Thomaz, and M. C. Fontoura Nogueira da Cruz, "Systemic alterations and their oral manifestations in pregnant women," Journal of Obstetrics and Gynaecology Research, vol. 43, no. 1, pp. 16-22, 2017.

[127] A. Hays, X. Duan, J. Zhu et al., "Down-regulated Treg cells in exacerbated periodontal disease during pregnancy," International Immunopharmacology, vol. 69, pp. 299-306, 2019.

[128] I. Cetin, P. Pileri, A. Villa, S. Calabrese, L. Ottolenghi, and S. Abati, "Pathogenic mechanisms linking periodontal diseases with adverse pregnancy outcomes," Reproductive Sciences, vol. 19, no. 6, pp. 633-641, 2012.

[129] J. Y. Kwak-Kim, H. S. Chung-Bang, S. C. Ng et al., "Increased T helper 1 cytokine responses by circulating $\mathrm{T}$ cells are present in women with recurrent pregnancy losses and in infertile women with multiple implantation failures after IVF," Human Reproduction, vol. 18, no. 4, pp. 767-773, 2003.

[130] S. Offenbacher, S. Lieff, K. A. Boggess et al., "Maternal periodontitis and prematurity. Part I: obstetric outcome of prematurity and growth restriction," Annals of Periodontology, vol. 6, no. 1, pp. 164-174, 2001.

[131] C. M. Cobb, P. Kelly, K. Williams, S. Babbar, M. Angolkar, and R. Derman, "The oral microbiome and adverse preg- nancy outcomes," International Journal of Women's Health, vol. Volume 9, pp. 551-559, 2017.

[132] M. González-Jaranay, L. Téllez, A. Roa-López, G. GómezMoreno, and G. Moreu, "Periodontal status during pregnancy and postpartum," PLoS One, vol. 12, no. 5, article e0178234, 2017.

[133] M. F. Fogacci, E. . O. C. Cardoso, D. . S. Barbirato, D. P. de Carvalho, and C. Sansone, "No association between periodontitis and preterm low birth weight: a case-control study," Archives of Gynecology and Obstetrics, vol. 297, no. 1, pp. 7176, 2018

[134] A. P. C. G. Seraphim, F. Y. Chiba, R. F. Pereira, M. S. . L. C. Mattera, S. A. S. Moimaz, and D. H. Sumida, "Relationship among periodontal disease, insulin resistance, salivary cortisol, and stress levels during pregnancy," Brazilian Dental Journal, vol. 27, no. 2, pp. 123-127, 2016.

[135] L. A. Daalderop, B. V. Wieland, K. Tomsin et al., "Periodontal disease and pregnancy outcomes: overview of systematic reviews," JDR Clinical \& Translational Research, vol. 3, no. 1, pp. 10-27, 2018.

[136] G. A. Foratori-Junior, B. M. da Silva, A. C. da Silva Pinto, H. M. Honório, F. C. Groppo, and S. H. de Carvalho SalesPeres, "Systemic and periodontal conditions of overweight/obese patients during pregnancy and after delivery: a prospective cohort," Clinical Oral Investigations, vol. 24, no. 1, pp. 157-165, 2020. 